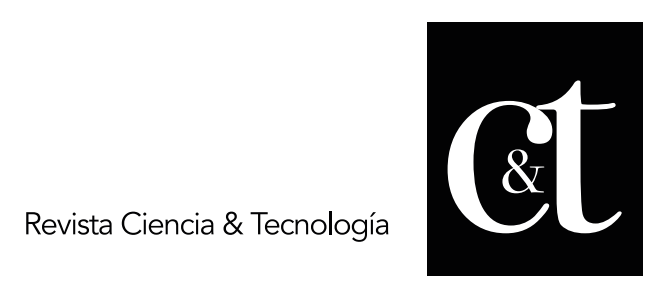

No. 32, 31 de octubre de 2021

ISSN impreso: 1390 - 6321

ISSN online: 2661 - 6734

\title{
Desarrollo e implementación de un sistema de luces de emergencia de uso doméstico
}

\section{Development and implementation of an emergency lighting system for domestic use}

\author{
Byron Oviedo ${ }^{1}$ \\ boviedo@uteq.edu.ec \\ http://orcid.org/0000-0002-5366-5917 \\ boviedo@uteq.edu.ec \\ Emilio Zhuma Mera² \\ ezhuma@uteq.edu.ec \\ http://orcid.org/0000-0002-3086-1413 \\ ezhuma@uteq.edu.ec \\ Eduardo Samaniego 3 \\ esamaniego@uteq.edu.ec \\ https://orcid.org/0000-0002-6196-2014
}

Recibido: 28/6/2021, Aceptado: 28/9/2021

\begin{abstract}
RESUMEN
Este proyecto está destinado al estudio de la seguridad de las personas ante situaciones de riesgo, específicamente en el diseño e implementación de un sistema de luces de emergencia para uso doméstico, primero se presenta la definición, importancia y el uso de cada uno de los componentes utilizados para el diseño e implementación del sistema, donde se muestran los diseños y construcción con sus respectivos materiales como microcontroladores, baquelitas, protoboards, sensores PIR, módulos ESP8266, NRF24L01 que se utilizaron para la construcción del mismo, finalmente se realizó el diseño y la simulación en el software PROTEUS, posteriormente se implementó un sistema de luces de emergencia para uso doméstico el cual consta de un temporizador, una unidad de control que se encarga de monitorear si es de noche y el flujo de energía eléctrica en el hogar, que envía la orden de encendido a los 2 unidades receptoras que se encargan de proveer iluminación, además el proyecto está diseñado para optimizar los recursos por lo que las unidades receptoras cuentan con sensores de proximidad que permiten detectar la presencia de personas y si se cumplen las 3 condiciones (que no haya energía eléctrica, que sea de noche y haya alguien cerca) las luces se encenderán permitiendo de esta forma mejorar la movilidad de las personas, también cuenta con un algoritmo que permite enviar mensajes
\end{abstract}

\footnotetext{
${ }^{1}$ Doctor, Universidad Técnica Estatal de Quevedo, Ecuador

${ }^{2}$ Magíster, Universidad Técnica Estatal de Quevedo, Ecuador

${ }^{3}$ Magíster, Universidad Técnica Estatal de Quevedo, Ecuador
} 


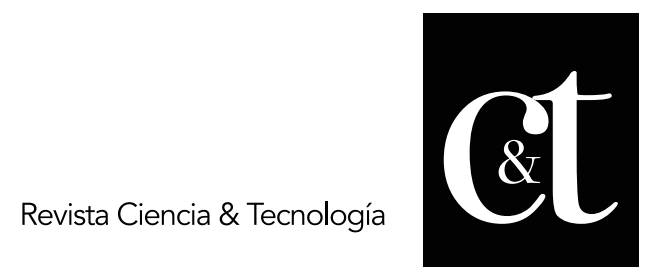

No. 32, 31 de octubre de 2021

ISSN impreso: 1390 - 6321

ISSN online: 2661 - 6734

mediante Twitter para notificar al usuario que el flujo de energía eléctrica en la noche se ha interrumpido.

Palabras clave: Sistema de luces, luces de emergencias, Microcontroladores, sensores PIR

\begin{abstract}
This project is intended to study the safety of people in risky situations, specifically in the design and implementation of an emergency lighting system for domestic use, first the definition, importance and use of each of the components is presented. used for the design and implementation of the system, where the designs and construction are shown with their material contents such as microcontrollers, bakelites, protoboards, PIR sensors, ESP8266 modules, NRF24L01 that were used for the construction of the same, finally the design and the simulation in the PROTEUS software, later an emergency lighting system for domestic use was implemented which consists of a timer, a control unit that is in charge of monitoring whether it is at night and the flow of electrical energy in the home, which sends the order of ignition to the 2 receiving units that are in charge of providing lighting, in addition the project is designed for o optimize resources so that the receiving units have proximity sensors that allow detecting the presence of people and if the 3 conditions are met (there is no electricity, it is at night and there is someone nearby) this way to improve the mobility of people, also has an algorithm that allows to send Twitter messages to notify the user by means of the flow of electricity at night has been interrupted.
\end{abstract}

Keywords: Light system, emergency lights, Microcontrollers, PIR sensors

\title{
Introducción
}

Hoy en día los sistemas de iluminación de emergencia son de suma importancia en nuestra vida, ya sea en el ámbito laboral, estudiantil, social, etc. Sirven para brindar apoyo a los usuarios ante cortes de energía eléctrica imprevistos, de una manera muy rápida y eficaz (Morales y Alvarado, 2014).

Esto es muy importante hoy en día ya que vivimos en una sociedad con tecnología de punta la misma que cada vez permite mejorar las condiciones de vida de la gente y en este caso incluso pueden salvarlas. Además están las de niveles de seguridad para las personas (Valdez, 2010), y las condiciones de ahorro de energía y búsqueda de energías limpias (Guevara y Lara, 2015). Actualmente, los sistemas de iluminación de emergencia están presentes en casi cualquier lugar, desde empresas, universidades, colegios hasta escuelas, pero a pesar de la relativa facilidad que existe para poder implementar estos sistemas de iluminación de emergencia aún no se cuenta con ellos para su uso doméstico de forma compacta y que permita mayores funcionalidades que la iluminación estáticade los sistemas industriales. La demanda eléctrica de los hogares puede verse como un ítem vital y puede leerse en un contexto mayor para asumirlo como referencial (Gálvez, Mariel y Hoyos, 2016). 


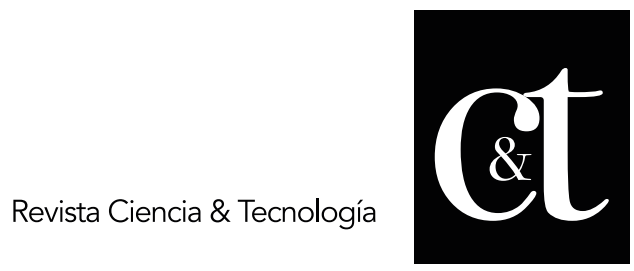

No. 32, 31 de octubre de 2021

ISSN impreso: 1390 - 6321

ISSN online: 2661 - 6734

Es por esta razón que el presente proyecto tiene como finalidad la implementación de un sistema de luces de emergencia totalmente adaptado para el uso doméstico, que permita a los usuarios mayores funcionalidades que los actuales sistemas de uso industrial, haciendo uso de los conocimientos adquiridos en las unidades de aprendizaje impartidas en este módulo así como de las unidades de aprendizaje de módulos anteriores.

La falta de un sistema de luces de emergencia de uso doméstico es un tema al que debe brindársele atención y buscarle una solución de forma oportuna debido a que, existen diversos factores que producen un corte de energía eléctrica en los hogares, desde catástrofes naturales hasta simples fallos en el sistema eléctrico. El problema se agudiza durante la noche ya que el ojo humano tarda de 3 a 4 minutos en adaptarse parcialmente a la oscuridad permitiendo así al menos distinguir sombras y evitar choques, sin embargo este tiempo no es suficiente para actuar de forma oportuna durante una situación de riesgo en la cual el factor visión juega un papel primordial. Asimismo, se busca eficiencia en la proyección de energía (García y González, 2020).

En la industria existen diversas normas a seguir en caso de emergencias, como por ejemplo señalización reflectiva, rutas de evacuación previamente definidas, puntosde encuentros y luces de emergencias, esta última permite tener una visión completa de los objetos a nuestro alrededor y así tener una mejor respuesta ante catástrofes, un ejemplo de ello son los terremotos los cuales generalmente causan fallas en el sistema eléctrico elevando la tasa de mortalidad si se producen por las noches, ya que al no poder ver aumenta el pánico y con ello el riesgo de sufrir accidentes.

Los hogares generalmente no poseen algún sistema de iluminación de emergencia, ante cortes de energía, motivo por el cual es necesario un sistema económico, compacto, de bajo consumo y con poco impacto en la estética del hogar (Hancevic y Navajas, 2015). El presente proyecto encuentra su justificación basado en que en cualquier momento puede ocurrir alguna catástrofe o fenómeno que afecte al suministro eléctrico dentro de un hogar, inclusive el mal uso de un equipo o electrodoméstico puede causar este corte en la energía eléctrica y si esto llegase a ocurrir durante lanoche dejaría a las personas que se encuentren en el lugar en total oscuridad, buscando una manera para iluminarse con el objetivo de recuperar visibilidad parcialo en casos extremos tener que abandonar rápidamente el lugar sin correr riegos dechocar con algún obstáculo o no hallar la salida.

Dicho esto, el motivo por el que este proyecto toma de referencia a los sistemas deiluminación de emergencia utilizados en el sector industrial (Cavassa, 2010), y buscala manera de adecuarlo para el uso doméstico, es para poder brindar la posibilidadde contar con un sistema de iluminación de emergencia en los hogares, que permitaa los usuarios moverse con tranquilidad ante un corte de energía eléctrica inesperado. Gracias a los avances tecnológicos y conocimientos adquiridos sobre el tema se puede realizar un trabajo óptimo, implementando equipos autónomos (con batería incorporada) y de acuerdo a como se desarrolle la parte electrónica queden programados para reaccionar ante una situación establecida (en nuestro caso la ausencia de energía eléctrica e iluminación), obteniendo como resultado un sistemaconfiable, más aún si se trata de un equipo de seguridad. 


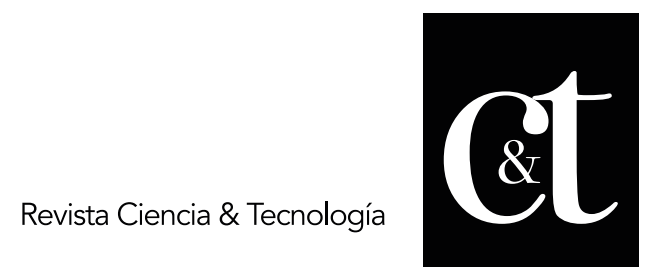

No. 32, 31 de octubre de 2021

ISSN impreso: 1390 - 6321

ISSN online: 2661 - 6734

\section{Métodos}

\section{Fase 1: Investigación Preliminar}

Primero se procedió a buscar un PIC que cumpla con los parámetros necesarios que permitan la interacción de forma sencilla con un transceptor NRF24L01 para lacomunicación de la parte emisora con las receptoras. El pic seleccionado es el PIC16F876A el cual posee los pines y el protocolo de comunicación serial (SPI) necesarios para el empleo en las luces de emergencias domésticas. (Parera, 2016)

El amplio uso de las baterías AA en los hogares del mundo y su bajo costo las hace propicia para ser la fuente de alimentación del prototipo, dándole a los dispositivos remotos la independencia necesaria para aprovechar la comunicación serial.

La línea de energía eléctrica y la de servicios de internet son diferentes lo cual hace posible que la funcionalidad de enviar un mensaje en twitter de advertencia de queha habido un apagón sea realizable, siempre y cuando el router doméstico posea una fuente de energía de respaldo como un regulador UPS.

Existe apagones que ocurren a altas horas de la noche, horas en que los habitantes de las casas se encuentran en estado de sueño por lo cual no son necesarias la activación de las luces de emergencia ya que esto implica un gasto innecesario de energía de las baterías.

El tiempo para mantener encendidas las luces debe ser lo suficiente para salvaguardar la integridad físicas de las personas y permitirles encontrar otras mejores fuentes de iluminación o la salida, y procurar no malgastar la energía de laspilas de alimentación.

\section{Selección de Materiales}

Para la elaboración del proyecto de luces de emergencia se procedió a seleccionar los siguientes elementos: (Salvador Martínez García, 2016)

- PIC 16F876A

- Transceptores NRF24L01

- Osciladores de cristal XT-4Mhz

- Panel de 24 LEDs

- Capacitores de 22uF

- Transistores 2N2222A

- Led Azul

- Pulsadores

- Protoboards

- Baterías AA 1.5v

- Quemador - Programador

- Temporizador NE555

- Resistencias

- Condensadores

- Cables de cobre

- Diodos Zener 
- Switch (2 estados)

- Porta Baterías

- Módulo WIFI ESP8266

- Sensor de movimiento PIR HC-SR501

- Fotorresistencia LDR

\section{Fase 2: Diseño de la unidad de control y monitoreo.}

\section{Diseño de temporizador}

El tiempo de funcionamiento de la activación de las luces leds se definió cerca de los 3 minutos, lo cual se supone es suficiente para cumplir con los objetivos del producto, para ello se usó una configuración de un temporizador 555 como monoestable calculando el valor de la resistencia y capacitor mediante la página http://gzalo.com/555/ definiéndolas en $4.7 \mathrm{M}$ ohns y 47 uF respectivamente.

\section{Distribución de voltaje}

Para el diseño del circuito se utilizó la aplicación de software "Proteus" tomando como eje central el microcontrolador y las conexiones a sus pines, empezamos con el emisor (unidad de control) conectando el pin 20 a un voltaje positivo y a tierra enel pin 8.

Hay que recalcar que la alimentación que llega al microcontrolador fue reducida desu valor inicial de $6 \mathrm{~V}$ hasta llegar a $4.5 \mathrm{~V}$ con el uso de Diodos zeners empleados como reguladores de baja de tensión, además de dividir el voltaje en otra subparte de $3 \mathrm{~V}$ para emplearlo a la alimentación del transceptor y del módulo ESP8266. Para habilitación del PIC hay que proporcionarle una alimentación de $5 \mathrm{~V}$ a través de una resistencia de $10 \mathrm{~K} \Omega$ al pin 1 (MCLR-reset).

Al usar un oscilador externo de cristal de $4 \mathrm{Mhz}$, se debe conectar entre los pines 9 y 10 del microcontrolador a un condensador cerámico de $22 \mathrm{pF}$, con lo cual servirá de reloj para nuestro dispositivo.

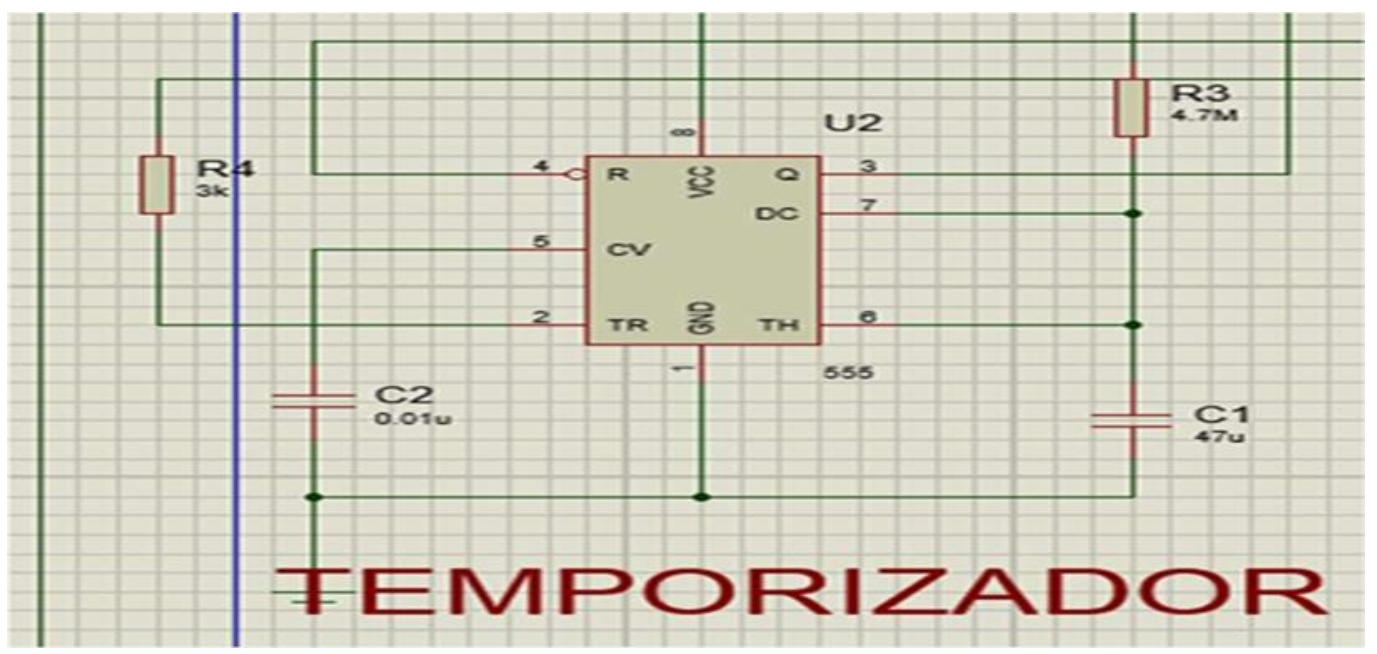

Figura 1. Diseño del circuito temporizador Fuente: Elaboración propia 


\section{Monitoreo de Energía eléctrica}

Se empleó un transistor con las siguientes conexiones: La base a una respectiva resistencia que sigue al cargador de la base, el emisor a tierra y el colector al pin 25(RB4). La fotocelda está en serie con una resistencia como divisor de voltaje y en paralelo al pin 2 (RA0). El pulsador fue conectado al pin 26 (RB5) con su resistenciainterna.

\section{Conexión de Transceptor NRF24L01}

Se conectó el transceptor usando el puerto $C$ de la siguiente manera: La alimentación usando la división inicial del voltaje de $3 \mathrm{~V}$ y la respectiva tierra, la funcionalidad CCN al pin 12 (RC1), CE al pin 13 (RC2), SCK al pin 14 (RC3), MISO al pin 15 (RC4) y MOSI al pin 16 (RC5).

En el transistor aquí las conexiones cambian, quedando de la siguiente manera: Labase a una respectiva resistencia al pin 4 (RA2), el emisor a tierra y el colector a latierra de las luces.

\section{Conexión de Módulo WIFI ESP82666}

El módulo ESP8266 es un módulo de bajo consumo pero variable, llegando incluso a picos de $250 \mathrm{~m}$ (amp) durante le comunicación inalámbrica lo que puede causar un reseteo total del módulo y por consiguiente que no se logré enviar el mensaje. (Donate, 2012).

Para las conexiones del módulo el pin VCC y CH_PD deben conectarse a 3 voltios, se utiliza el pin GPIO2 como entrada digital y va conectado al pin RA5 del pic.

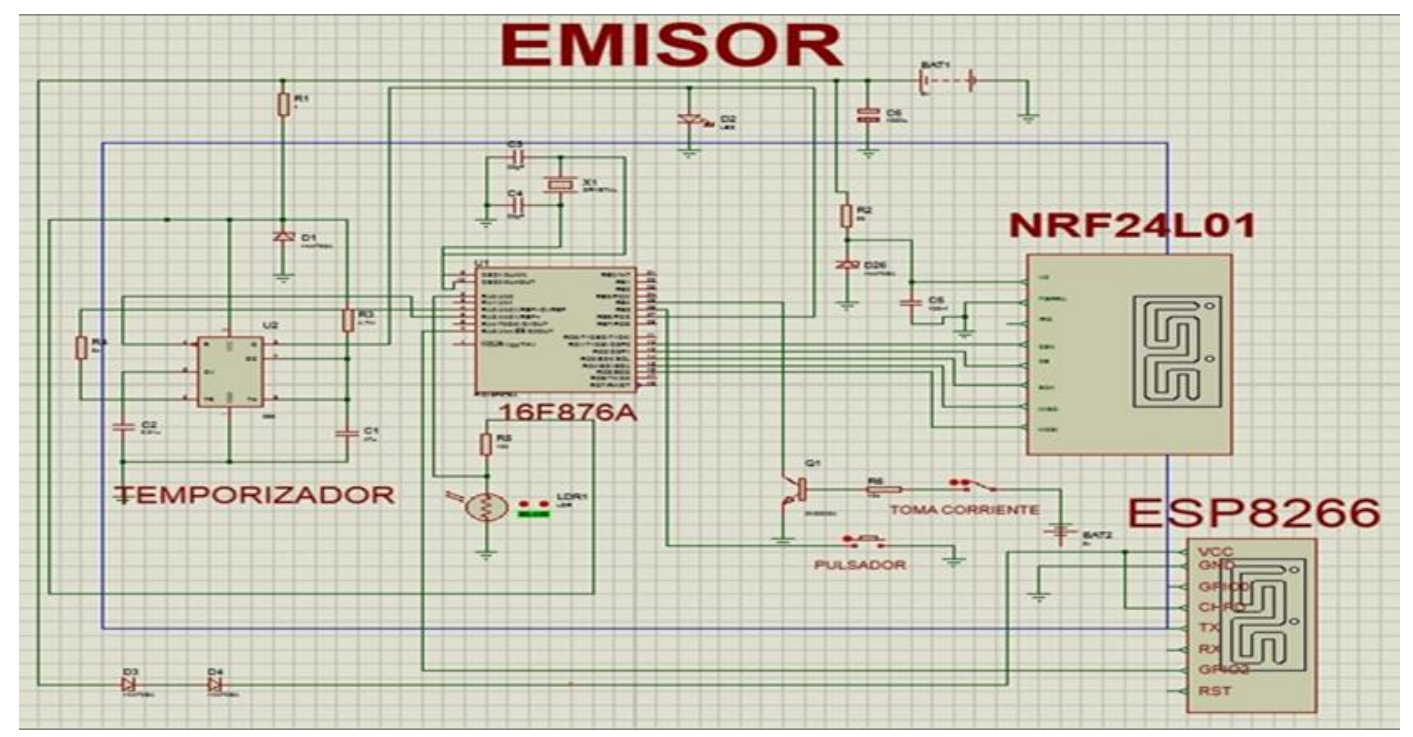

Figura 2. Diseño del circuito emisor (Unidad de control)

Fuente: Elaboración propia 


\section{Fase 3: Diseño de las unidades remotas de iluminación Diseño de circuito receptor}

El diseño del circuito receptor comparte mucha similitud con el circuito emisor, comola conexión del oscilador de cristal y alimentación con la diferencia que para la regulación de voltaje en este caso se usó 2 diodos en serie para hacer una caída de tensión de $1.8 \mathrm{~V}$ y a tierra fue designado el pin 19. En el transceptor también essimilar pero se le añadió una función extra que es la de la IRQ al pin 21 RB0.

\section{Implementación del sensor PIR}

El sensor de movimiento PIR se alimenta con 5 voltios y su salida digital es de $3.3 \mathrm{v}$ por lo cual se conectará esta al pin RB5 y cuando se mande la señal de encendido estará atento de que haya movimiento para posteriormente encender la luces. (Areny, 2013).

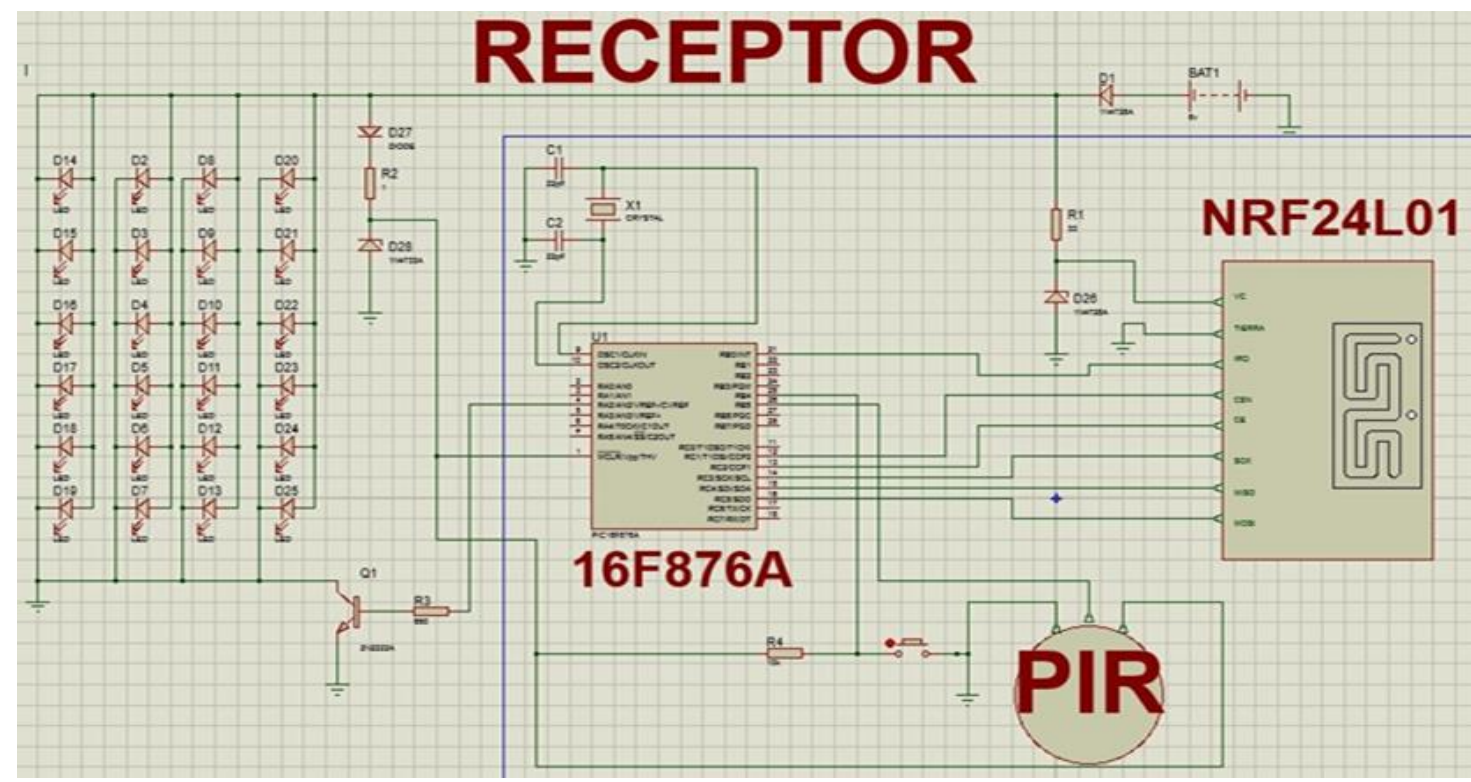

Figura 3. Diseño del circuito receptor

\section{Programación de microcontroladores}

El desarrollo del código se llevó a cabo para responder correctamente al hardware previamente diseñado, para facilitar su creación se usó el compilador PIC CCS el cual emplea el lenguaje $\mathrm{C}$.

Con el fin de optimizar el uso de las baterías el microcontrolador está programado para permanecer en modo SLEEP (muy bajo consumo) sólo saliendo de este modo mediante una interrupción externa indicativa de que se ha caído la energía eléctrica en el hogar o alguno otra interrupción ha existido.

La comunicación entre el microcontrolador y el módulo NRF24L01 se facilitó con el uso de la librería lib_rf2gh4_10.h propia para este propósito. 


\section{Programación de Módulo ESP8266}

La programación se realizó mediante IDE de arduino 1.6.7, añadiendo la librería ESP8266 y usando la plataforma con el mismo nombre. Lo cual le permite trabajar con total independencia del microcontrolador.

En su código se guarda una página web en HTML la cual se puede acceder conectándose al punto de acceso que tiene como nombre "Luces De Emergencia VII" con la contraseña TELEMATICAVII y abriendo en el navegador la dirección 192.168.4.1, de esta forma se puede actualizar la SSID y la contraseña de nuestra red local con conexión a internet. Está contraseña y nombre de red se almacenan en la EEPRON del módulo ESP8266 por lo cual no es necesario actualizar dichos datos al reiniciar

Si se ha detectado el pulso bajo en GPIO2 y se tiene conexión a internet se procederá a publicar el mensaje en twitter el cual tendrá el siguiente formato:

\section{Luces De Emergencia @LucesTelVII}

Figura 4. Módulo ESP8266: Tweet

Fuente: Elaboración propia

Se utilizó el servicio de publicación de tweets de thingspeak disponible para aplicaciones de internet de las cosas, facilitando el envío del mensaje.

\section{Resultados}

\section{Prueba e Implementación del circuito electrónico}

Se procedió a armar el circuito físicamente, basado en el diseño realizado, para proceder a ejecutar las pruebas pertinentes para la verificación de su correcto funcionamiento en protoboards.

\section{Realización e implementación de prototipo}

Para ahorrar espacio y hacerlo lo más compacto posible, se desarrolló el circuito en baquelitas perforadas de acuerdo al diseño realizado en Proteus, ubicando los componentes en la mejor posición posible, verificando después de cada punto que exista conexión y que no se produzca ningún corto (conexión no deseada) con otro punto adyacente. 
No. 32, 31 de octubre de 2021

ISSN impreso: 1390 - 6321

ISSN online: 2661 - 6734

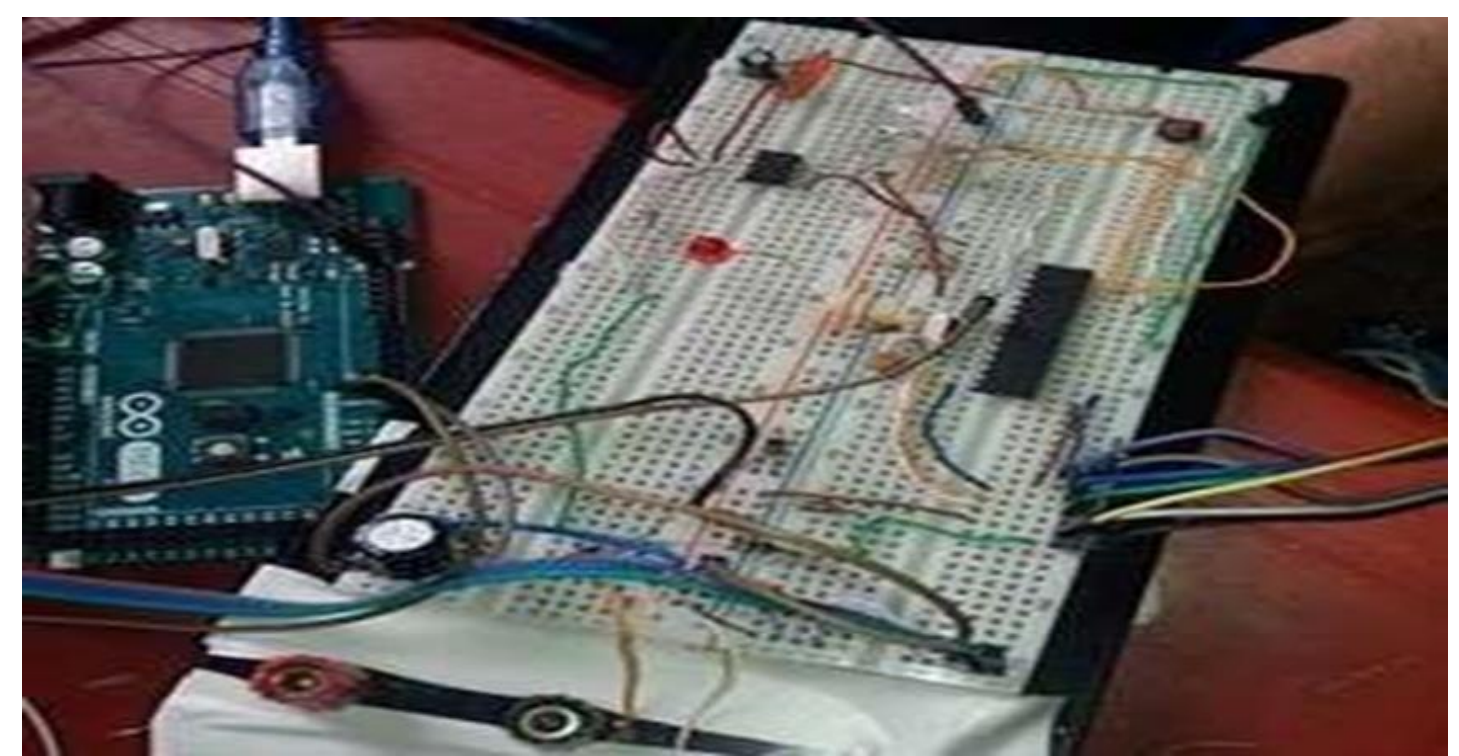

Figura 5. Unidad de control

Fuente: Elaboración propia

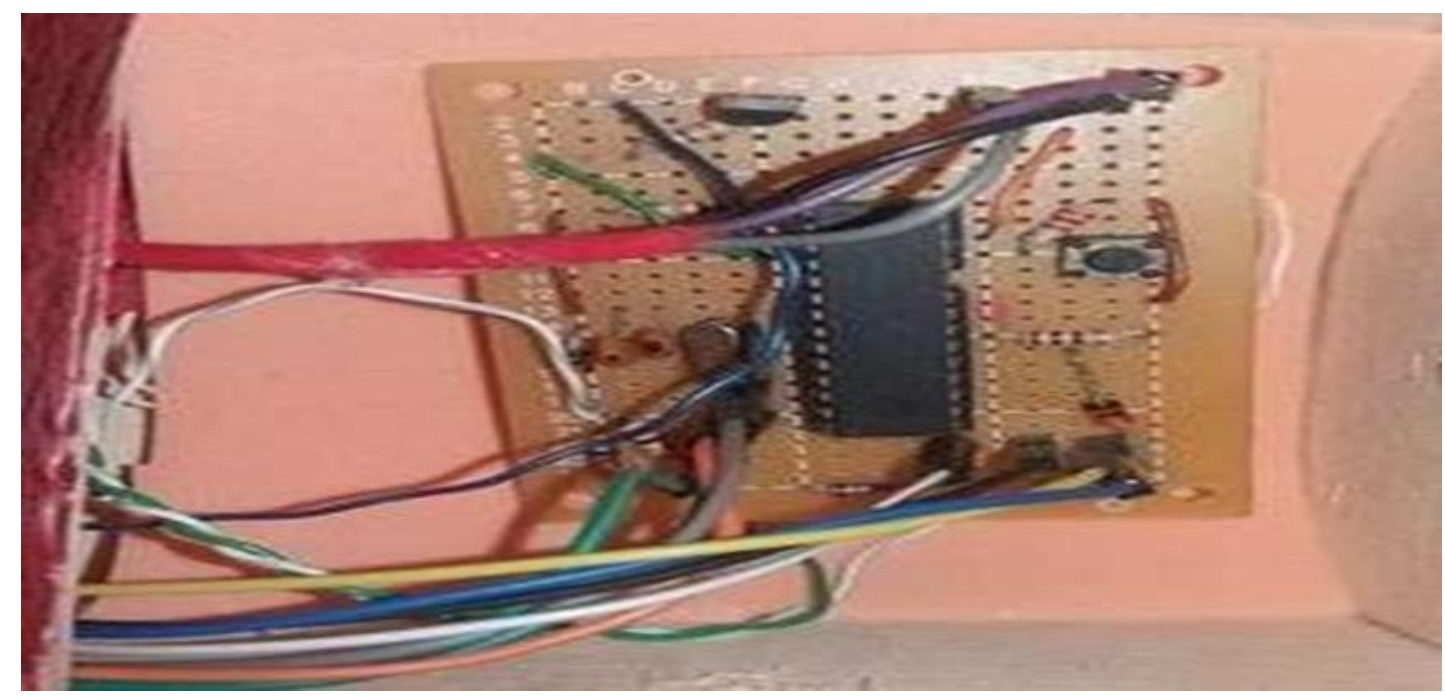

Figura 6. Unidad receptora

Fuente: Elaboración propia 


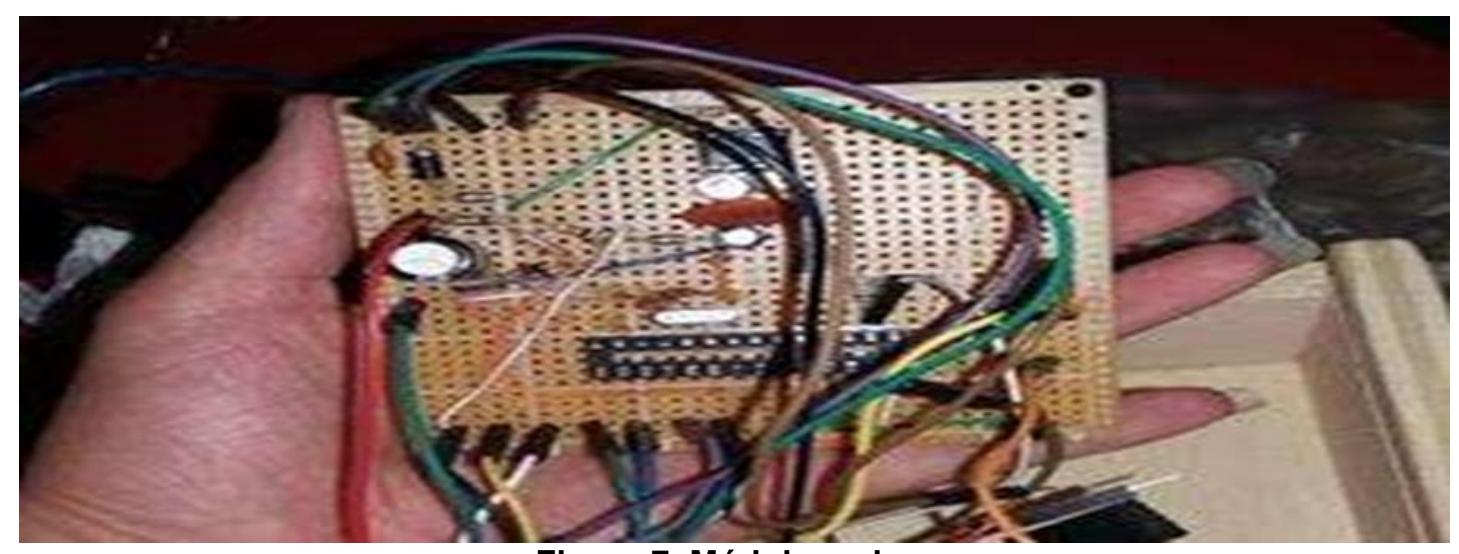

Figura 7. Módulo emisor Fuente: Elaboración propia

Posteriormente se ubicó el circuito dentro de un case elaborado en madera el cual dispone de 2 compartimentos internos, uno para las baterías y otro para el circuito mismo, dejando orificios que facilitan la salida de los módulos NRF24L01, ESP8266 así como el interruptor de encendido y fotocelda.

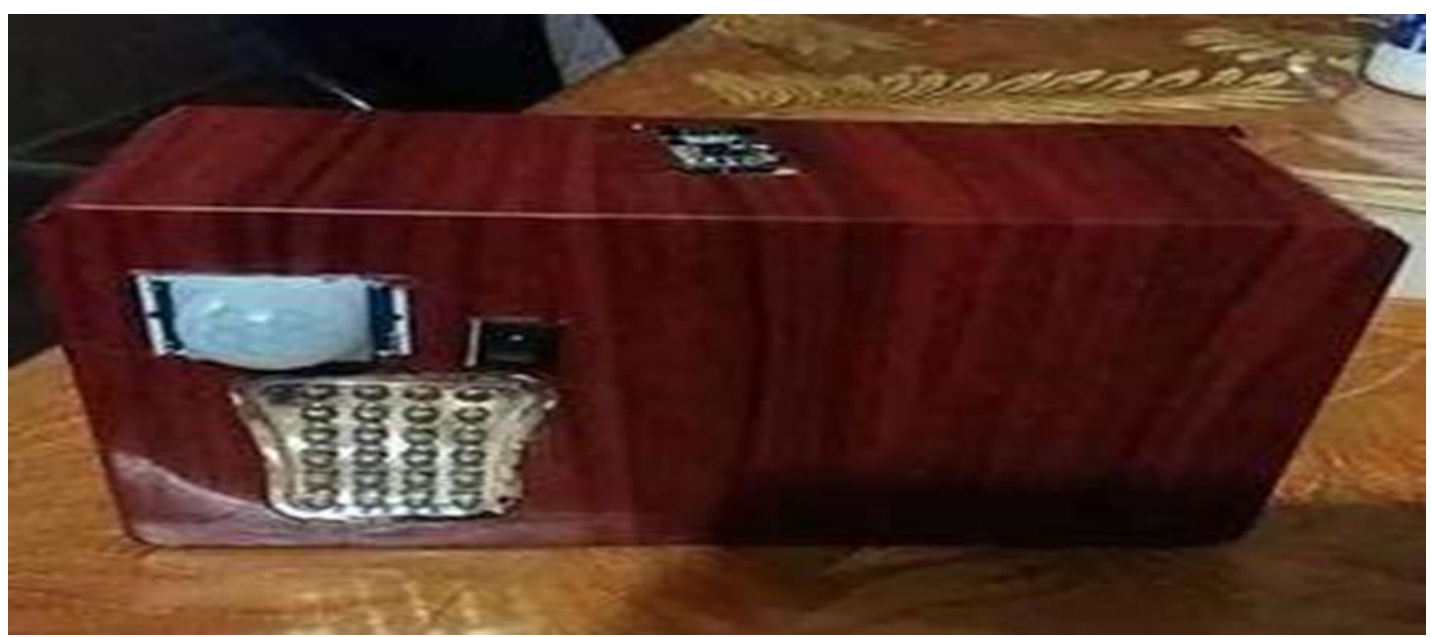

Figura 8. Sistema implementado

Fuente: Elaboración propia 


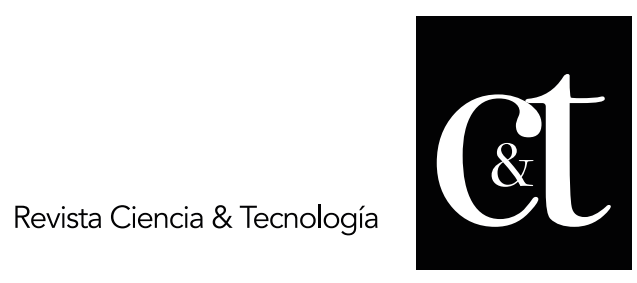

No. 32, 31 de octubre de 2021

ISSN impreso: 1390 - 6321

ISSN online: 2661 - 6734

\section{Dimensión: Disponibilidad}

El sistema de luces de emergencia para uso doméstico cumple con los requerimientos de disponibilidad al trabajar sin problema alguno en todo el tiempo que se encuentre activo, solo no estará disponible en los tiempos de baja por mantenimiento los mismos que son programados previamente para no afectar a los usuarios de este.

\section{Dimensión: Escalabilidad}

Es factible para el sistema de luces de emergencia de uso doméstico, apropiarse del concepto de escalabilidad, ya que al tratarse de un sistema formado por componentes electrónicos, permitirá con la implementación de nuevos componentes incluir nuevas funcionalidades al sistema.

\section{Dimensión: Utilización}

Debido a su diseño el sistema de luces de emergencia para uso doméstico, es totalmente utilizable, incluso para personas que son usuarios noveles de equipos electrónicos, la facilidad de aprender cómo utilizarlo radica en que es un sistema compacto, que no requiere mayor esfuerzo tanto para su instalación como para su uso.

\section{Dimensión: Eficiencia}

Mediante la realización de las pruebas se comprobó el correcto funcionamiento del sistema, brindando así, iluminación a la zona del hogar donde se encuentren ubicadas las unidades receptoras pudiendo ser estas, escaleras, salas 0 habitaciones permitiendo a las personas poder reaccionar de forma adecuada ante un corte de energía eléctrica imprevisto, mejorando la movilidad de las mismas.

\section{Conclusiones}

Es posible adaptar un sistema de iluminación de emergencia industrial a uno para el uso doméstico, haciendo uso de los conocimientos de electrónica básica y programación de microcontroladores adquiridos en módulos anteriores.

Los componentes electrónicos que se utilizaron fueron microcontroladores PIC 16F876A, Transceptores NFR24L01, Sensores PIR y un módulo WiFi ESP8266, a más de los componentes básicos como resistencias capacitores y transistores.

Se decidió agregar al sistema de luces de emergencia para uso doméstico como nueva funcionalidad la capacidad de detectar la presencia de una persona mediante los sensores PIR, y la capacidad de enviar mensajes de notificación a los usuarios vía Twitter indicándoles que ha habido un corte de energía eléctrica en la noche.

El desarrollo del sistema de luces de emergencia para uso doméstico se realizó cumpliendo con las necesidades de iluminación de los usuarios y al mismo tiempo permite ahorrar recursos al contar con sensores PIR que permitirán que se active la iluminación solo si una persona está cerca. 


\section{Referencias bibliográficas}

Areny, R. P. (2013). Sensores y acondicionadores de señal. Barcelona, España: Macrocombo.

Cavassa, C. R. (2010). Seguridad Industrial. México: Limusa.

Gálvez, P., Mariel, P. y Hoyos, D. (2016). Análisis de la demanda residencial de los servicios básicos en España usando un modelo QUAIDS censurado. Estudios de Economía vol.43 no.1 Santiago jun.

García, E. González, A. (2020). Solución de iluminación eficiente energéticamente para una vivienda sustentable. Ingeniería Eléctrica. vol.41 no.2 La Habana mayo-ago.

Guevara, A. y Lara, J. (2015). Mitigación del cambio climático a través de un alumbrado público eficiente en México: superando los retos políticos en aras de la eficiencia económica y el equilibrio ambiental. Acta universitaria. vol.25 no.1 México ene./feb.

Hancevic, P. y Navajas, F. (2015). Consumo residencial de electricidad y eficiencia energética. Un enfoque de regresión cuantílica. El trimestre económico. vol.82 no.328. México oct./dic.

Donate, A. H. (2012). Electrónica Aplicada. Barcelona, España: Macrocombo. Parera, A. M. (2016). Electrónica básica en automoción. México: Boixareu.

Morales, D. y Alvarado, A. (2014). Análisis del consumo de energía eléctrica domiciliaria en Tampico, Tamaulipas. CienciaUAT vol.8 no.2 Ciudad Victoria ene-jun.

Salvador Martínez García, J. A. (2016). Electrónica de potencia: componentes, topologías y equipos. (Thomson, Ed.) España.

Valdez, R. (2010). La seguridad industrial como un factor intrínseco de la competitividad. Perspectivas, núm. 25, enero-junio, pp. 153-168. 